\title{
Molecular Diffusion Water Exchange in Compartments of Tissue Water of Maize
}

\author{
Iryna ZAITSEVA ${ }^{1, a^{*}}$, Volodymyr SYROVATKO ${ }^{2, b}$ \\ ${ }^{1}$ Oles Honchar Dnipropetrovsk National University, Dnipropetrovsk, Ukraine \\ ${ }^{2}$ Institute of Soil Protection of Ukraine, Kyiv, Ukraine \\ airinza_Idfr@mail.ru, brodgrunt_dp@i.ua
}

Keywords: Zea mays, isotope technique, tritium, water exchange, symplast, apoplast

\begin{abstract}
For studying water exchange in compartments of tissue water of maize, kinetic curves of dilution of tritium labeled water $\left({ }^{3} \mathrm{H}\right)$ were used, with the incubation of plant tissues in it. By resolving the summary kinetic curves into components, we determined the constants of membranes' permeability for exchange diffusion flux in two compartments of tissue water - membranerestricted water and water of higher mobility in free space of cell envelopes, and quantitative content of symplastic and apoplastic water in the plant tissues. Significant differences of rates of water-exchange processes in the symplast and apoplast of maize types with various genotypes were found at the temperatures of $20,30,40$ and $50^{\circ} \mathrm{C}$. In the Pioneer hybrid these figures vary in the intervals of $1,83-3,67 \mathrm{~s}^{-1} \cdot 10^{-4}$ and $0,91-1,33 \mathrm{~s}^{-1} \cdot 10^{-3}$, whereas in self-pollinated line A-204 the intervals are 1,80-3,51 and 1,12-1,48 accordingly. Peculiar features of water exchange reactions suggest the higher physiological constancy of the Pioneer hybrid under thermal action.
\end{abstract}

\section{INTRODUCTION}

Physiological processes in plants are largely dependent on the amount and condition of water in tissues and cells. It is known that water in the plant cell represent poly-fraction system where each fraction differs from the other one by the strength of bond with biopolymers of the cell. Staying in continuous motion, water molecules form a part of unstable aggregates which are generated at the expense of hydrogen bonds existing within $10^{-11} \mathrm{~s}$ only, or they run into free state with the duration limited by electrostatic interactions between molecules [1].

The part of intracellular (or symplastic) water in the aggregated (bound) state is not uniform with bio-colloids and cell ions with regard to bond strength [2,3,4]. Weakly bound water is represented by water of diffusion layers of hydration shells and structured water in the area of ion action, which serves as a solvent. Strongly bound water is a part of the first hydration layer around the ion, and it cannot be a solvent for the substances of intracellular space [5,6]. Unbound intracellular water with higher mobility can be slightly polarized under action of ions and generally its characteristics have little difference from those of pure water. Water of vacuoles which is the largest by volume should be referred to the compartment of symplastic water as well. Unbound and poorly aggregated vacuole water still features low activity, since plasmatic membrane (tonoplast) restricts its availability for exchange processes.

As distinct from symplastic water, apoplastic water remained in free space of cell walls is the least bound and the most mobile (labile) water in water-exchange processes. A part of this water is held by hydrogen bonds with the hydrophilic groups of macromolecules, however the bulk of it in turgid cells is kept owing to surface tension in free interfibrillar spaces of the cell wall $[7,8]$. Therefore, free water fraction in the plant cell is represented mainly by apoplastic water.

In recent years, the issue on the role in the plant body of various water fractions defined as "bound" and "free" water is of particular relevance because water-retaining properties of plant tissues and, as a whole, drought and overeating resistance of plants depend on the degree of structuring and aggregation of water molecules $[9,10]$.

The method of labeled water as one of the most promising and accurate methods for examination of water exchange in plants was used in this work for studying the tissue water 
compartmentation $[11,12,13]$. The method is based on the capacity of tissue water to exchange fully with tritiated water ${ }^{3} \mathrm{H}^{1} \mathrm{H}_{2} \mathrm{O}$ of the outer solution [14,15]. The time required for achievement of the equilibrium distribution of isotopes varies in the wide range (from 10 to $100 \mathrm{~min}$ ) and depends on the species of plants and organs which tissues are incubated in the labeled solution [14]. During this time it is possible to trace the kinetics of isotope dilution of the labeled solution with tissue water. Mathematical analysis of kinetic curves allows determining the compartmentation of water in tissues under study and parameters which characterize molecular water exchange of individual fractions of water and its energy characteristics.

\section{MATERIAL AND METHODS}

Objects of investigations were maize plants of the Pioneer hybrid and self-pollinated line $A$ 204 which were chosen in order to identify the peculiar features of water exchange, depending on genetic properties of plants. The plants were cultured in the standard aqueous nutrient medium, i.e. $0,164 \%$ aqueous solution of the main macro- and micro-elements equalized by $\mathrm{pH}$ value and quantity of cations. Plants after having reached the phase of the third leaf were used for the experiment.

Compartmentation of water was determined in stems, for which purpose they were cut into segments of 6-7 mm long. Samples of plant tissue obtained by this method ( $m=2 \mathrm{~g}$ ) were poured with $4 \mathrm{ml}\left(4 \cdot 10^{-3} 1\right)$ of tritium-containing water ${ }^{3} \mathrm{H}^{1} \mathrm{H}_{2} \mathrm{O}$ (outer solution) with the specific radioactivity of $3,1 \cdot 10^{5} \mathrm{~Bq} / \mathrm{ml}$ and incubated in thermostat at temperatures of $20,30,40$ or $50^{\circ} \mathrm{C}$. In order to achieve maximum homogeneity in distribution of tritium water in the outer solution, magnetic mixer was placed into the reaction vessel.

In the definite intervals of time (from 1 to $150 \mathrm{~min}$ ) samples of the outer solution were taken by micro-syringe. For reducing the error associated with the change in volume of the outer solution during sampling, the volume of sample to be taken should be minimal, but sufficient to measure the radioactivity of the solution. These conditions are satisfied by the volume of sample equal to $V=8$ $\mathrm{mcl}\left(8 \cdot 10^{-6} 1\right)$. In total, in each variant of the experiment 20-25 samples were taken of 160-200 mcl in total volume, i.e. $4-5 \%$ of the initial volume of the outer solution, which minimized the systematic error of the experiment.

On completion of the experiment the plant tissues were dried to the constant weight, weighed and total initial amount of tissue water $m$ was calculated. In each variant, the experiment was carried out in 3 replications.

Specific radioactivity of tritium in samples taken from the outer solution was determined with the use of the "Ultrabeta-1210" liquid scintillation counter. For the time of exposure (during 1 $\min$ ) about $1,5 \cdot 10^{5}$ decompositions occurred in the volume of $8 \mathrm{mcl}$; even taking into account the gradual dilution of the outer solution with tissue water it is quite sufficient for recording on the counter. In accordance with the change in value of the specific radioactivity in time, kinetic curves of isotope dilution were plotted and analyzed by the method of logarithmic decomposition [16,17].

\section{RESULTS AND DISCUSSION}

Based on the experiments, we obtained the curves of kinetics dilution of the outer solution using water of stems of the hybrid and self-pollinated maize types in the conditions of various temperature modes. The example of typical kinetic curves is given in Fig. 1.

Logarithmic representation of data allows allocating two segments of the curve: nonlinear segment (in the initial period of incubation) and linear segment, when starting from a certain point of time the logarithmic dependence becomes linear one. For the example with the Pioneer hybrid shown in the figure (Fig. 2) at the temperature of $20^{\circ} \mathrm{C}$ this time is equal to about $50 \mathrm{~min}$, at $30^{\circ} \mathrm{C}-$ $35 \mathrm{~min}$. 

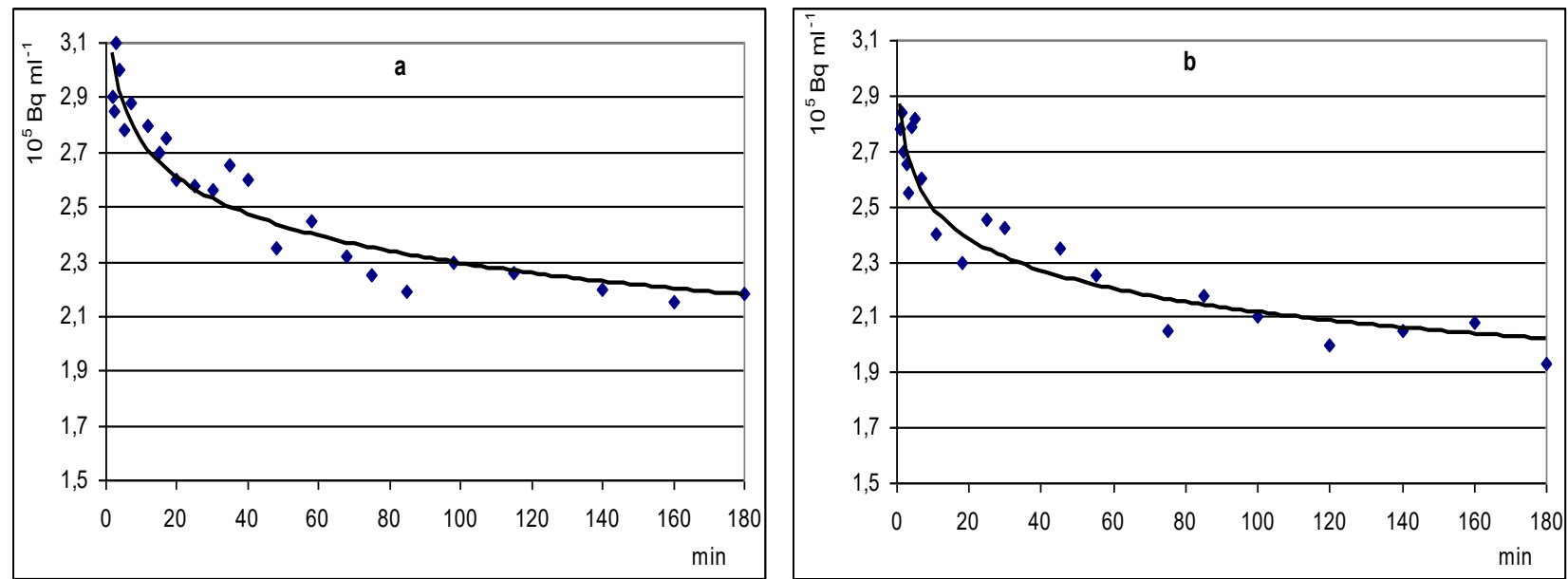

Fig. 1. Experimental kinetic curves of isotope dilution of tritium-containing outer solution with tissue water of the Pioneer hybrid. a. $20^{\circ} \mathrm{C} \mathrm{b} .30^{\circ} \mathrm{C}$
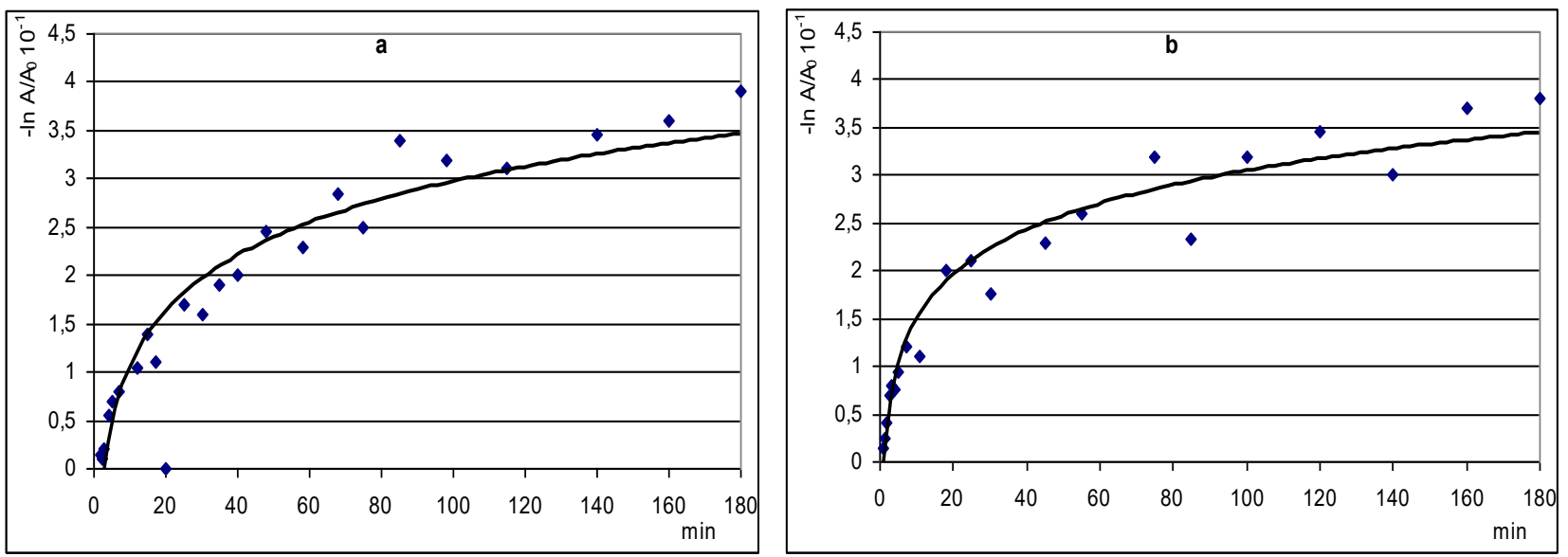

Fig. 2. Logarithmic representation of the curves of isotope dilution of the outer solution. On OY axis in the expression $-\ln \frac{A}{A_{0}} 10^{-1}: A_{0}$ - initial specific activity of the outer solution, $A$-activity at the present moment. a. $20^{\circ} \mathrm{C} \mathrm{b.} 30^{\circ} \mathrm{C}$

Therefore, kinetic curve of exchange reactions is represented by the sum of two exponential terms, where each of them reflects the diffusion water exchange in the definite compartment - being quicker in one of them and slower in the other:

$$
m(t)=\sum_{i=N}^{1} m_{i}\left(1-e^{-k_{i} t}\right),
$$

where $m(t)$ - total weight of exchanged water in the incubated tissues at the present moment; $m_{i}-$ mass of water in the $i$-th compartment; $k_{i}$ - kinetic constant of water exchange of the $i$-th compartment.

Upon reaching the equilibrium distribution of water of the outer solution and plant tissues, the equation below is fulfilled

$$
m=m_{0}\left(A_{0} / A_{\infty}-1\right),
$$

where $A_{0}$ - initial specific activity of ${ }^{3} \mathrm{H}$ in the outer solution; $A_{\infty}$ - activity of the solution after reaching of tritium equilibrium distribution in the system; $m_{0}, m$ - mass of water in the outer solution and tissues, accordingly.

Using the experimental values of specific activity of the outer solution before placement of the plant tissue into it and after reaching the equilibrium distribution of water, according to equation (2), we can calculate the total mass of tissue water. 
In this case, mass of exchanged water at any moment of time $t$ in the interval from the incubation start $t_{0}$ to the equilibrium distribution of water $t_{\infty}$ is described by the equation

$$
m(t)=m_{0}\left(A_{0} / A(t)-1\right) \text {. }
$$

Dividing equation (3) by (2), we obtain the equation for the calculation of part of the exchanged water at the given moment of time $t$ :

$$
m(t) / m=\left[A_{0} / A(t)-1\right] /\left[A_{0} / A_{\infty}-1\right] .
$$

If the constants $k_{i}$ in equation (1) satisfy the condition $k_{i}<<k_{i+1}$, i.e. tissue water is divided into compartments by the degree of its availability for exchange, then, starting from the definite moment of time, all exponential terms except the first one will be actually unchanged over time. It means that by the time when still minor changes occur in slowly exchanging compartment, water of the second compartment actually exchanges with the outer solution in full. This being the case, after taking a logarithm of equation (1) we obtain a ratio:

$$
-\ln \left(1-\frac{m(t)}{m}\right)=-\ln \frac{m_{1}}{m}+k_{1} t
$$

where $m$ - weight of all compartments of tissue water, i.e. $\sum_{i=N}^{1} m_{i}$.

Consequently, using the equations (4) and (5), as well as the experimental curves of changing of tritium specific activity in the outer solution, it is possible to calculate parameters of water exchange of the first compartment. In this algorithm the compartment with the lowest water exchange rate having the minimal value of kinetic constant $k_{i}$ (which can be designated as $k_{1}$ ) is calculated first. Accordingly, the calculated mass of water in this compartment is designated as $m_{1}$. If we consider the process of water exchange in time, this first "slow compartment" which contains various forms of bound (symplastic) water starts to exchange with the outer solution lastly, after complete water exchange of "quick" apoplastic compartment.

Example of the dependence $-\ln \left(1-\frac{m(t)}{m}\right)$ on time calculated in accordance with equation (5) is given in Fig. 3. Based on the form of dependence, it is possible to note, similarly to chart in Fig. 2, that starting from the definite moment of time (time of stoppage of water exchange of "quick" compartment of free water) the curve assumes strictly linear shape. The intersection of this straight line of the axis of ordinates determines the fixed term of equation $-\ln m_{1} / m$, of which it is possible to calculate the mass of slow compartment of bound water $m_{1}$ in total mass of tissue water $m$. Angle of inclination of the straight line determines the constant of water exchange rate of this compartment $\left(k_{1}=\operatorname{tg} \alpha\right)$.

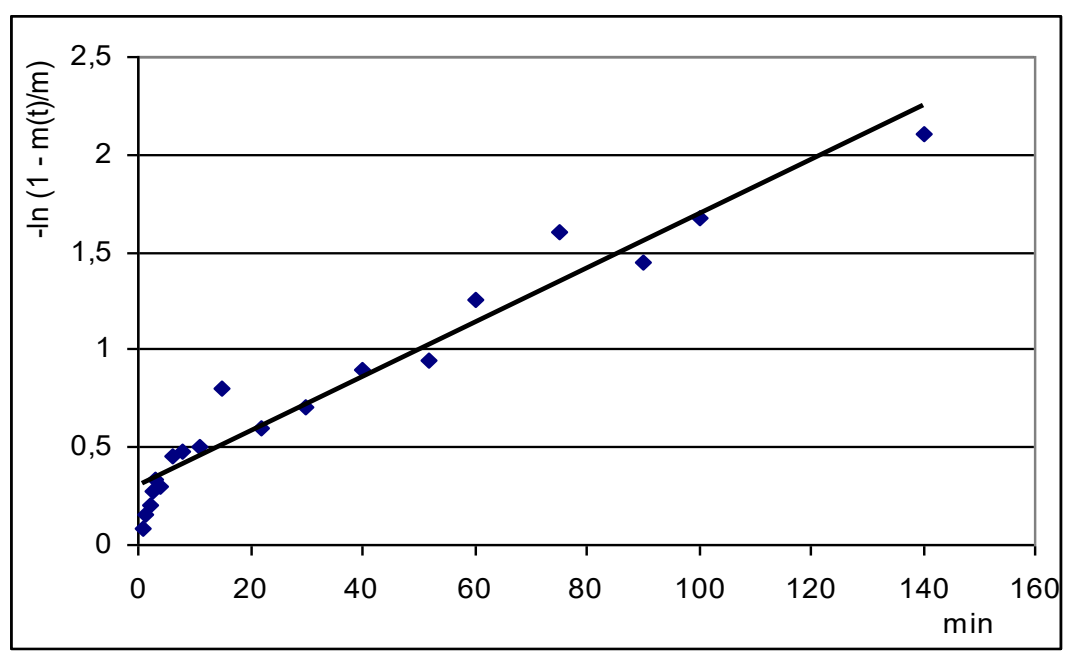

Fig. 3. Logarithmic representation of total curve of the mass of tissue water in the process of its exchange with the outer solution (the Pioneer hybrid, temperature of $50^{\circ} \mathrm{C}$ ) 
For the further decomposition of total kinetic curve it is necessary to calculate the amount of exchanged water in the first compartment $m(t)$ at all points of time $t$, when samples were taken in the experiment. We used for this the values $m_{1}, k_{1}$ calculated according to equation (5) and equation (1), rearranged for one compartment:

$$
m_{1}(t)=m_{1}\left(1-e^{-k_{1} t}\right) .
$$

After gradual subtraction of this term from the total mass of exchanged tissue water at each point of time we obtain a new dependence similar to equation (5):

$$
-\ln \left(1-\frac{m_{2}(t)}{m-m_{1}}\right)=-\ln \frac{m_{2}}{m-m_{1}}+k_{2} t
$$

This dependence describes water exchange of the second (quick) compartment, and it turns out to be significant only within the short interval of time after the incubation starts, until all tissue water exchanges for the outer solution, and it is clearly seen in Fig. 4. Using the ratio (7), we have calculated parameters of the second compartment of tissue water - its mass $m_{2}$ and constant of water exchange rate $k_{2}$.

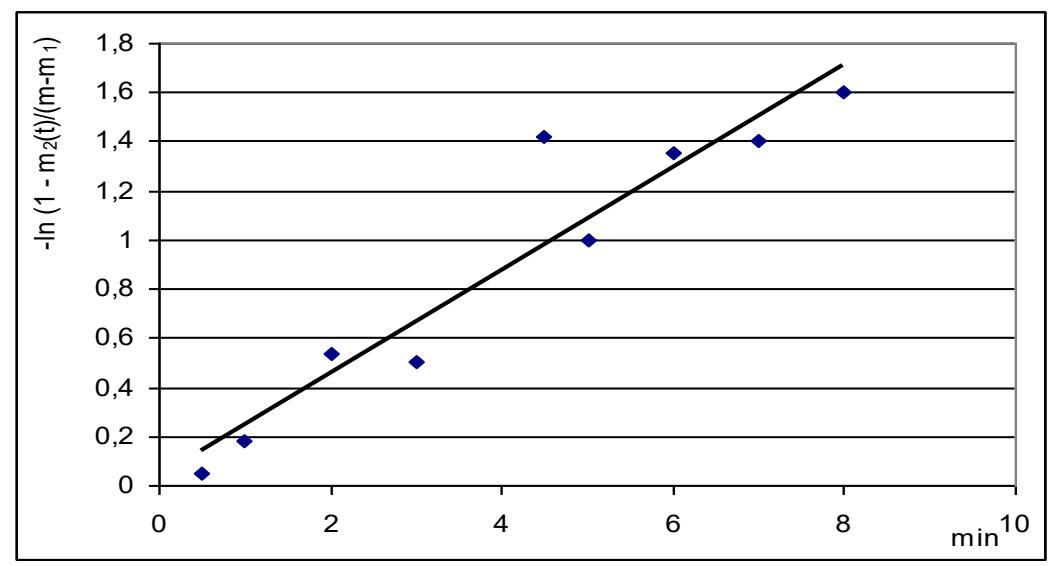

Fig. 4. Logarithmic representation of dynamics of water exchange of the second (quick) compartment of tissue water (the Pioneer hybrid, temperature of $50^{\circ} \mathrm{C}$ )

When using the described algorithm, it is possible to continue decomposition of the experimental curve, by means of allocation of compartments with varying rate of water exchange, until free term disappears (Fig. 3).

Usage of this method for analyzing the curves of isotope exchange of the labeled solution with tissue water in maize stems allowed to distinguish two compartments and to calculate their parameters (Table 1). The first compartment corresponding to the constant $k_{1}$, evidently, should be identified with membrane-restricted water, i. e. symplast water, which gradually exchanges with the outer solution. The second one corresponding to the constant $k_{2}$ is represented by water of free space of cell walls and intercellular spaces, i. e. water of the apoplast.

Table 1. Quantitative content of symplastic water and constant of rates of water exchange reactions of the symplast and apoplast

\begin{tabular}{|c|c|c|c|c|}
\hline $\begin{array}{c}\text { Object of } \\
\text { research }\end{array}$ & $\begin{array}{c}\text { Tempera- } \\
\text { ture, }{ }^{\circ} \mathrm{C}\end{array}$ & $k_{1}\left[\mathrm{~s}^{-1} \cdot 10^{-4}\right]$ & $k_{2}\left[\mathrm{~s}^{-1} \cdot 10^{-3}\right]$ & $m_{1}[\%]$ \\
\hline \multirow{3}{*}{ Pioneer hybrid } & 20 & $1,83 \pm 0,125$ & $0,91 \pm 0,065$ & $71 \pm 3,4$ \\
\cline { 2 - 5 } & 30 & $2,66 \pm 0,124$ & $1,08 \pm 0,061$ & $77 \pm 4,2$ \\
\cline { 2 - 5 } & 40 & $3,17 \pm 0,103$ & $0,83 \pm 0,069$ & $67 \pm 4,8$ \\
\cline { 2 - 5 } & 50 & $3,67 \pm 0,250$ & $1,33 \pm 0,062$ & $68 \pm 5,3$ \\
\hline \multirow{3}{*}{$\begin{array}{c}\text { Self-pollinated } \\
\text { line } A-204\end{array}$} & 20 & $1,80 \pm 0,173$ & $1,12 \pm 0,053$ & $52 \pm 5,3$ \\
\cline { 2 - 5 } & 30 & $2,30 \pm 0,143$ & $1,21 \pm 0,049$ & $64 \pm 7,1$ \\
\cline { 2 - 5 } & 40 & $2,97 \pm 0,081$ & $1,02 \pm 0,057$ & $51 \pm 6,5$ \\
\hline
\end{tabular}


The results show that hybrid and self-pollinated maize forms significantly differ by the content of water in the symplast and apoplast. For example, quantitative content of symplastic water with regard to the apoplastic water in the hybrid tissues is noticeably higher in various temperature conditions, making from 67 to $77 \%$ of total water content in cells. In self-pollinated line the tissue water is distributed actually in equal proportions $(49-64 \%)$ between two basic compartments. This ratio is maintained at various temperatures as well. Consequently, in maize types differing by their genotype the index of water compartmentation and its distribution between the protoplast and the cell wall is quite stable, and varies only slightly under the influence of elevated temperatures. If we consider the conditions of overheating and drought, which often affect the cultivated plants in Steppe zone of Ukraine, the degree of adaptability of this index is higher in the hybrid, since the most of tissue water is maintained in the bound or membrane-restricted state.

With the maintenance of general patterns of water compartmentation typical for various maize types, it is necessary to note certain peculiarities of the impact of high temperatures on this process. It is found that the response to temperature stress in the hybrid and in the line is manifested synchronously - at $30^{\circ} \mathrm{C}$ in both types the fraction of bound water increases at the expense of reduction of free water quantity (Fig. 5), that can be regarded as the primary nonspecific response to the action of super-optimal factor.

With the further rise in temperature the process is reversed - the share of free apoplastic water which is more subject to the negative influence of dehydrating forces increases. This process can be connected with the disturbance of spatial structure of bio-colloids and destruction of intermolecular bonds with involvement of water under action of high-temperature stress.
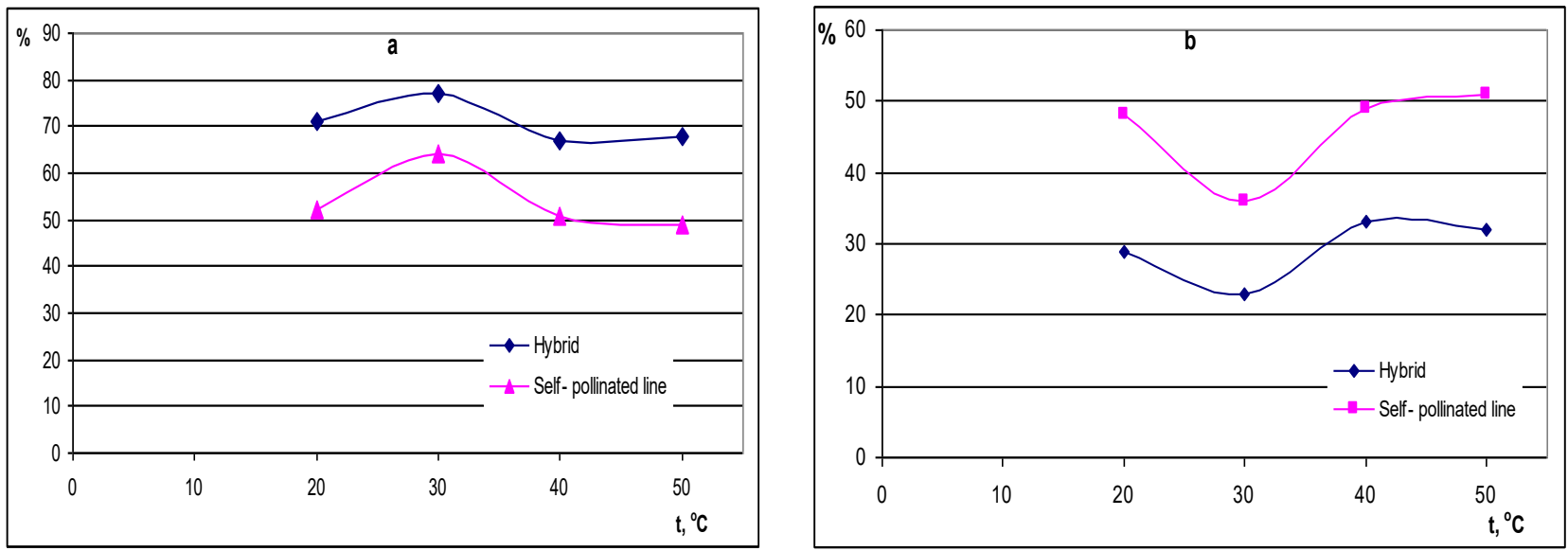

Fig. 5. Temperature dependence of quantitative content of maize tissue water compartments. a. Symplastic water b. Apoplastic water

Analysis of water exchange constants (Table 1) shows that for maize types under study the higher rate of water-exchange reactions in the apoplast is typical; it about 10 times exceeds this index in the symplast. At the same time, maize types varying in genotype slightly differ by the value of this index. In self-pollinated line the rate of water exchange in the apoplast is higher, while in the symplast it is lower compared to the hybrid, and this ratio is maintained at various temperature conditions as well. Taking into account the fact that the primary acceptor of stress factor action is free water, higher mobility of its molecules in self-pollinated line may result in the development of water deficit. High content of bound water in the hybrid even at the increased rate of water exchange ensures its higher resistance to the temperature stress.

It should be noted that the character of dynamics of changes in the rate of water exchange depending on the temperature factor for both maize types under study is similar, just as it is observed with regard to water content in the compartments. Besides, the rate of water exchange in the apoplast agrees well with the changes in the content of apoplastic water (Fig. 5, b) - when the rate increases the amount of water in apoplast is falling, whereas the decrease in the rate to the minimum at $40^{\circ} \mathrm{C}$ is accompanied by growth of the water mass in the apoplast to the maximum. 
Temperature dependence of the rate of water exchange in the symplast for two maize types is the linear one, and in the conditions of weak effect of temperature $\left(30^{\circ} \mathrm{C}\right)$ the considerable growth of water exchange rate is accompanied by the increase in the amount of membrane-restricted water (Fig. 5, a). This discrepancy, apparently, can be explained by activation of the adaptive intracellular mechanisms, as a rule, energy-dependent ones [18], at the first stage of stress action aimed at the increase in water structuring and maintaining of metabolism at the optimal level.

\section{CONCLUSIONS}

As a result of study of water-exchange reactions of the hybrid and self-pollinated maize line by the method of isotope dilution of labeled ${ }^{3} \mathrm{H}^{1} \mathrm{H}_{2} \mathrm{O}$ water and analysis of resulting data by progressive decomposition of exponential curves, quantitative parameters of two compartments of the tissue water (mass and rate of water exchange) have been established. The hybrid is characterized by higher content of membrane-restricted water and low rate of exchange reactions of water in free space of cell walls; in general, it is the adaptive physiological characteristic which increases the resistance to dehydrating environmental factors. Inverse ratios of these indices in selfpollinated line give evidence of its lower physiological constancy.

In the conditions of overheating, similar pattern of changes of parameters of water exchange in maize types under study is manifested. The content of symplastic water is growing as an adaptive response to the moderate thermal effect. Under action of sublethal temperatures the rate of waterexchange reactions in the apoplast and symplast is growing sharply, because of high permeability and structural-functional disturbances of membranes.

Identity of dynamics of changes of water compartmentation and water exchange rate in the hybrid and self-pollinated forms of maize in response to the temperature stress is the evidence of generality of adaptation mechanisms which have formed in the process of Zea mays phylogenesis, and they do not depend on the degree of the genotype heterozygosis.

\section{References}

[1] Kramer P.J., Bayer J.S. Water relations of plants and soils. Orlando: Acad. Press. 1995.

[2] Weatherley P.E. The state and movement of water in the leaf. Symp. Soc. Exper. Biol. 19(2) (1965) 157-167.

[3] Velicanov G.A. Vacuolar symplast and methodological approach to monitoring water self diffusion between vacuoles of contacting root cells. 54(5) (2007) 683-692.

[4] Anisimov A.V., Suslov M.A. and Alyab`ev A.Y. Symplasmic transport of water along the roots depends on pressure. Russian Journal Plant Physiology. 61(4) (2014) 512-519.

[5] Fricke W. Water movement between epidermal cells of barley leaves - a symplastic connection. Plant, Cell Environ. 23 (2000) 991-997.

[6] Martnoia E., Maeshima M. and Neuhaus H.E. Vacuolar transporters and their Essential role in plant metabolism. J. Exp. Bot. 58 (2007) 83-102.

[7] Boller T., Wiemken A. Dynamics of vacuolar compartmentation. Annu. Rev. Plant. Physiol. 37 (1986) 137-164.

[8] Steudle E. Water transport across roots. Plant Soil. 167 (1994) 79-90.

[9] Ionenko I.F., Anisimov A.V. Effect of water deficit and membrane destruction on water diffusion in the tissues of maize seedling. Biol. Plant. 44 (2001) 247-252.

[10]Kudoyarova G.R., Veselov D.S. and Kholodova V.P. Current state of the problem of water relations in plants under water deficit. Russian Journal Plant Physiology. 60(2) (2013) 165-175. 
[11]Sedykh N.V., Stupishina E.A. Investigation of water and water exchange state of a plant cell by IR absorption spectra. Physiol. and Biochem. of Cult. Plants, Kiev. 4(3) (1972) 295-299.

[12]Zakharin A.A. A method for the investigation of fast kinetics of plant water exchange when affected by changes in the external osmotic pressure. Russian Journal Plant Physiology. 48(1) (2001) 143-149.

[13] Van der Weerd L., Claessens M.M.A.E, Efde C. and Van Ash. Nuclear magnetic resonanse imaging of membrane permeability changes in plant during ocmotic stress. Plant Cell Environ. 25 (2002) 1539-1549.

[14]Lenskyi L.A. Tritium in water-retaining systems. M.: Energoizdat. 1981.

[15] Shmatko I.G., V.A. Syrovatko. Labeled water transport in plants, in: Regulatory mechanisms of physiological processes in plants. Naukova dumka, Kiev. (1985) 26-29.

[16]Zaitseva I.O., Povorotnaja M.M. Phenotypic variability of plant leaves of Acer genus, introduced into steppe zone of Ukraine. Visn. Dnipropetr. Univ. Ser. Biol. Ecol. 22(2) (2014) 133-144.

[17]Zaitseva I.O. Dynamics of water exchange processes of species of Acer L. genus in connection with their drought resistance. Visn. Dnipropetr. Univ. Ser. Biol. Ecol. 12(1) (2004) 54-62.

[18]Heber U., Wiese C., Hedrich R., Savchenko G. and Bukhov N.G. Energy-dependent solute transport from the apoplast into the symplast of leaves during transpiration. Russian Journal Plant Physiology. 49(1) (2002) 32-43. 